\title{
Preface
}

\section{Special Issue on Mathability}

The concept of mathability was introduced in 2013 as a branch of cognitive infocommunications that investigates combinations of artificial and natural cognitive capabilities relevant to mathematics, including a wide spectrum of areas ranging from low-level arithmetic operations to high-level symbolic reasoning. In its definition, it was pointed out that investigations on mathability extend to the question of how artificial mathematical capabilities can be quantified. An important goal of mathability is to develop a set of methodologies, which can be applied to emulate and enhance human mathematical capabilities.

During the last years, questions connected to this concept have been investigated by several scientists. The topics considered and discussed include, among others, connections between mathability and computer assisted methods in mathematics, the relation of some human factors to mathability, possible quantifications associated with mathability, relationships between the mathability of devices and human mathematical abilities as well as cognitive and educational aspects of mathability (e.g. mathability and cognition, computer assisted education, spreadsheet tools, coaching in education, creative problem solving). More than 60 scientific papers published in prestigious scientific journals or conference volumes as well as several chapters or parts of books have been devoted to the presentation of these studies.

This special issue extends and enriches these results with a collection of some recent achievements in this field.

Attila Gilányi

Guest Editor 